\title{
Spontaneous compartment syndrome in a patient with hemophilia B
}

\author{
Joseph Levi ${ }^{1} \cdot$ Tara Stansbury $^{1} \cdot$ Matthew D. Marschall $^{1}$. Jamie Allen ${ }^{1} \cdot$ Andrew Steele $^{2} \cdot$ Lauren M. Crowley $^{1}$. \\ Kristine L. Schultz ${ }^{1} \cdot$ Kevin R. Weaver ${ }^{1} \cdot$ Yee Cheng Low ${ }^{2} \cdot$ Jeanne L. Jacoby ${ }^{1}$
}

Received: 8 January 2021 / Accepted: 19 February 2021 / Published online: 25 March 2021

(c) The Author(s), under exclusive licence to Canadian Association of Emergency Physicians (CAEP)/ Association Canadienne de Médecine d'Urgence (ACMU) 2021

Keywords Compartment syndrome $\cdot$ Hemophilia B $\cdot$ Spontaneous bleed $\cdot$ Emergency department

\section{Introduction}

Compartment syndrome is a serious condition that occurs when perfusion pressure falls below tissue pressure within a closed compartment. This can result from extrinsic forces, such as a tight cast, or intrinsic forces, such as excessive swelling, leading to increased interstitial fluid pressure. Delayed treatment can cause neurovascular compromise and may lead to permanent muscle and nerve damage, limb loss, rhabdomyolysis, and death [1]. Immediate consultation with the appropriate surgical service (Orthopedics, Plastic Surgery, General Surgery or transfer) depending on the facility and resources available should be conducted in unison with imaging and treatment as soon as the diagnosis is clinically suspected. Patients with all forms of hemophilia can develop intra-articular, intra-muscular, or neural hemorrhage with minimal or no trauma [2]. Hemophilia B is a rare X-linked genetic disorder, characterized by factor IX deficiency [3]. The incidence of Hemophilia B is 1 in 25,000 male births; Hemophilia A occurs in 1:5000 male births [4].

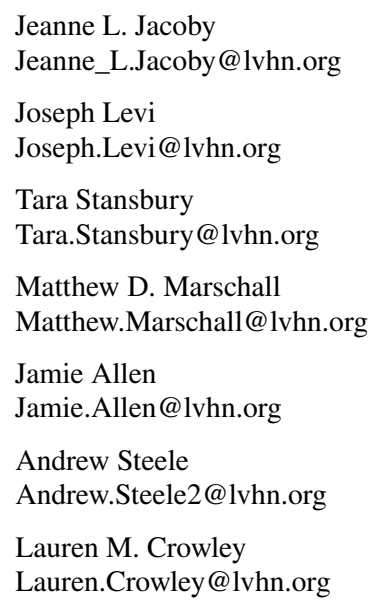

\section{Case}

A 38-year-old male emergency department (ED) patient presented with complaints of left forearm, wrist, and hand pain. His pain was diffuse and located from his elbow down to his fingers. He described it as sharp, severe, and rated 10/10. It was worse with any movement of the wrist, forearm, and elbow, and nothing made it better. Associated symptoms included forearm, wrist and fingers swelling, decreased range of motion and numbness/tingling mostly in digits 4-5. His symptoms started $24 \mathrm{~h}$ prior to his ED visit and had been gradually worsening. He denied any trauma to the extremity.

He had a past medical history of hemophilia B and hypertension. A brother also had hemophilia B. His vitals were only notable for a blood pressure of $229 / 145 \mathrm{mmHg}$. His physical exam was remarkable for left upper arm ecchymosis, diffuse swelling from the elbow to digits, and flexor posturing of ring and long fingers. His left flexor compartment was taut on clinical exam. He had decreased active and passive range of motion of his forearm (supination and pronation), wrist (flexion, extension, radial/ulnar deviation),

\author{
Kristine L. Schultz \\ Kristine_L.Schultz@lvhn.org \\ Kevin R. Weaver \\ Kevin_R.Weaver@1vhn.org \\ Yee Cheng Low \\ YeeCheng.Low@1vhn.org \\ 1 Department of Emergency and Hospital Medicine, Lehigh \\ Valley Health Network/University of South Florida Morsani \\ College of Medicine, Lehigh Valley Campus, Cedar Crest \\ Boulevard \& I-78, Allentown, PA 18103, USA \\ 2 Department of Surgery, Lehigh Valley Health \\ Network/University of South Florida Morsani College \\ of Medicine, Lehigh Valley Campus, Cedar Crest Boulevard \\ \& I-78, Allentown, PA 18103, USA
}


and digits 3-5 (held in flexion with decreased extension) due to pain. His anterior and posterior interosseous nerves and distal radial/ulnar strength were intact. His radial and ulnar pulses were intact. He had decreased sensation to light touch on the volar surface of the left hand and diffusely in digits 3-5. Compartment pressures were not measured prior to surgical consultation because the need for surgical intervention was evident.

A Hematology consult recommended $30 \mathrm{mg}$ of coagulation factor VIIa recombinant (NovoSeven) and obtaining a factor IX level. The patient was given labetalol for elevated blood pressure and fentanyl for pain. A clinical diagnosis of compartment syndrome was made; compartment pressures were not measured. His lab tests showed an initial hemoglobin $118 \mathrm{~g} / \mathrm{L}$, hematocrit $34.7 \%$, white blood cell count $0.01 \times 10^{9} / \mathrm{L}$., platelets $41,110^{9} / \mathrm{L}$ and normal INR of 1.0, Factor IX level was $<1 \%$ (reference $50-150 \%$ ) corresponding to severe hemophilia $B$. Patients with mild forms of hemophilia are more likely to present without a previous diagnosis of a bleeding disorder and compartment syndrome may be the initial presentation of hemophilia.

In the operating room, Plastic Surgery noted increased compartment pressures with bulging of the forearm compartment muscles upon release of the fascia. They performed ten left-hand decompression fasciotomies, a left carpal tunnel release, and two left forearm decompressive fasciotomies. Post-operatively, the patient was admitted to the intensive care unit for management of his hypertension and wound care. The patient's hemoglobin level was highest upon initial presentation $(11.8 \mathrm{~g} / \mathrm{dL})$ and dropped as low as $7.0 \mathrm{~g} / \mathrm{dL}$ during admission, requiring 8 units of packed red blood cell transfusions over the length of his stay.

Although his symptoms and hand function improved post-operatively, plastic surgery was unable to close his fasciotomy due to excessive swelling. He ultimately required skin graft placement on hospital day 16. Hematology continued to follow the patient throughout his stay and infused a total of $1544 \mathrm{mg}$ (50 times his initial dose) of NovoSeven over his 25-day admission. Upon discharge, he substantially improved. He had regained much of his forearm and wrist movement and sensation.

\section{Discussion}

Compartment syndrome is a serious condition that must be treated emergently. With increased compartment volume or increased interstitial fluid production, perfusion pressure may drop below that of the surrounding tissue and result in capillary collapse. Irreversible tissue injury may begin within $6 \mathrm{~h}$ of onset. The forearm is composed of three compartments, which typically have a pressure from 0 to $8 \mathrm{mmHg}$. Some common signs are hand and forearm paresthesias and pain out of proportion with passive digital extension [1]. Pressure measurement can be useful in diagnosing compartment syndrome; if the pressure is $>30 \mathrm{mmHg}$, compartment syndrome is likely. However, compartment syndrome remains a clinical diagnosis, and measuring pressures should not delay appropriate consultation and definitive management. As noted in this case where prompt surgical therapy was initiated and compartment pressures were not measured. Time to fasciotomy is inversely correlated with prognosis [5].

There are few isolated descriptions of spontaneous upper extremity compartment syndrome in the context of congenital coagulopathy in the published literature [6]. Those case studies emphasized replacing factors when hemophilia is recognized. Pre- and perioperative factor products should be given to minimize blood loss. Radiographs may be obtained to rule out bony injury, and ultrasound may be useful for evaluating blood flow, fluid collections, and swelling [2]. Three take-home points for the Emergency clinician are: first, that patients with all forms of hemophilia are at risk for compartment syndrome even in the absence of trauma; second, with increased compartment volume or increased interstitial fluid production, perfusion pressure may drop below that of the surrounding tissue and result in capillary collapse. Irreversible tissue injury may begin within $6 \mathrm{~h}$ of onset; third, surgical consultation should be made immediately when compartment syndrome is clinically suspected, in unison with imaging and medical treatment.

Acknowledgements The authors would like to acknowledge Erin Shigo, BA for assisting in the writing and formatting of this report.

Authors' contributions The authors all contributed to the formation of this manuscript.

\section{Declarations}

Conflicts of interest The authors declare that there is no conflict of interest regarding the publication of this article. The authors have no outside support information, conflicts or financial interest to disclose and this work has not been published elsewhere.

\section{References}

1. Broughton G. Compartment syndrome. In: Janis JE, editor. Essentials of plastic surgery, Italy. Quality Medical Publishing; 2006. p. 633-43.

2. Hoots K. Emergency management of hemophilia. In: Lee CA, Berntorp EE, editors. Textbook of hemophilia. Blackwell; 2010. p. 394-400.

3. Jentzsch T, Brand-Staufer B, Schäfer FP, Wanner GA, Simmen HP. Illustrated operative management of spontaneous bleeding and compartment syndrome of the lower extremity in a patient 
with acquired hemophilia A: a case report. J Med Case Rep. 2014;8:132. https://doi.org/10.1186/1752-1947-8-132.

4. National Organization for Rare Diseases. Hemophilia B. rarediseases.org. 2012. https://rarediseases.org/rare-diseases/hemop hilia-b/. Accessed 2 Feb 2021.

5. Olson SA, Glasgow RR. Acute compartment syndrome in lower extremity musculoskeletal trauma. J Am Acad Orthop Surg.
2005;13(7):436-44. https://doi.org/10.5435/00124635-20051 1000-00003.

6. Kim J, Zelken J, Sacks JM. Case report spontaneous forearm compartment syndrome in a boy with hemophilia A: a therapeutic dilemma. Eplasty. 2013;13:e16. 